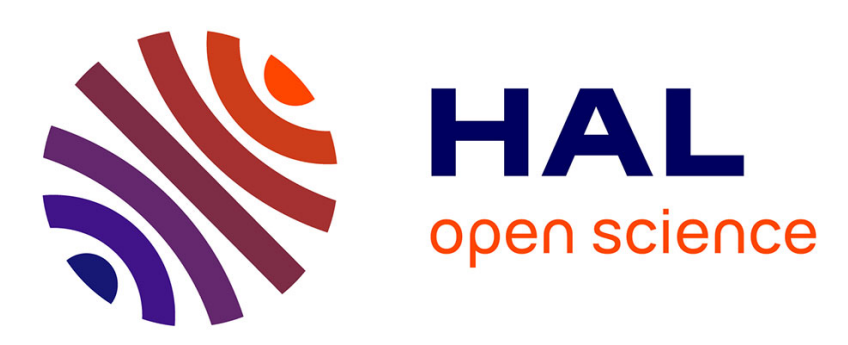

\title{
A micro-mechanics based strain gradient damage model: formulation and solution for the torsion of a cylindrical bar
}

Cécile Oliver-Leblond, H. Dumontet, D. Kondo

\section{- To cite this version:}

Cécile Oliver-Leblond, H. Dumontet, D. Kondo. A micro-mechanics based strain gradient damage model: formulation and solution for the torsion of a cylindrical bar. European Journal of Mechanics - A/Solids, 2016, 56, pp.19-30. 10.1016/j.euromechsol.2015.10.001 . hal-01223772

\section{HAL Id: hal-01223772 \\ https: / hal.sorbonne-universite.fr/hal-01223772}

Submitted on 3 Nov 2015

HAL is a multi-disciplinary open access archive for the deposit and dissemination of scientific research documents, whether they are published or not. The documents may come from teaching and research institutions in France or abroad, or from public or private research centers.
L'archive ouverte pluridisciplinaire HAL, est destinée au dépôt et à la diffusion de documents scientifiques de niveau recherche, publiés ou non, émanant des établissements d'enseignement et de recherche français ou étrangers, des laboratoires publics ou privés. 


\title{
A micro-mechanics based strain gradient damage model: formulation and solution for the torsion of a cylindrical bar
}

\author{
C. Oliver-Leblond ${ }^{\mathrm{a}, *}$, H. Dumontet ${ }^{\mathrm{a}}$, D. Kondo ${ }^{\mathrm{a}}$ \\ ${ }^{a}$ Institut Jean Le Rond d'Alembert (Sorbonne Universités, UPMC Univ Paris 06, CNRS, UMR 7190) \\ 4, Place Jussieu, 75252 Paris Cedex 05, France
}

\begin{abstract}
The present paper is devoted to the proposal of a theoretical formulation for an isotropic damage model with strain gradient. The approach is based on the non-local estimates of Drugan and Willis [1], exposed in terms of energetic methods, for the purpose of damage modelling. We first focus on the derivation of the non-local constitutive equations for the damage model which is fully analysed from the thermodynamics point of view. It is shown that the positive definiteness of the thermodynamic potential and the intrinsic dissipation are not ensured for every loading paths. The choice of the damage variable is briefly discussed. The equilibrium equation and the boundary conditions are presented. Then, the model is applied to the study of strain gradient torsion problem, for which the axi-symmetric solution is established. This allows us to study the size effect and to evaluate the impact of the non-local term on the damage evolution and the non-linear behaviour of the bar.
\end{abstract}

Keywords: Micro-mechanics, damage model, strain gradient

\section{Introduction}

In the context of durability studies of civil engineering buildings, damage mechanics offer an interesting framework to model the irreversible deterioration of quasi-brittle materials. However, when softening occurs, strain localisation can be observed and local damage theory becomes unreliable. From the numerical point of view, the loss of objectivity implies a dependency between the amount of dissipated energy and the mesh refinement. Non-local damage models are widely used to overcome those issues and can be split into three different categories: regularisation of the strain variable as in the integral non-local model [2] or in the implicit gradient model $[3,4]$, introduction of the damage gradient in the strain energy $[5,6,7]$ or introduction of the strain gradient in the strain energy [8].

Another important motivation of nonlocality is the need to capture size effects observed in fracture experiments on concrete [9]. Indeed, the size of the fracture process zone is independent of the size of the structure - provided it does not interfere with its boundaries - and therefore it can be observed that the nominal strength of geometrically similar specimens is dependent on the structure size. A characteristic length, related to the size of the fracture process zone, is needed to describe the transitional type of size effect. A review on nonlocality, including an historical summary of its main motivations can be found in $[10]$.

These regularised models introduce additional material parameters whose calibration can be difficult to carry out. Indeed, the identification of these material parameters cannot be achieved through simple experiments due to the localisation of the fields, which generally introduce structural effects in the mechanical response. Moreover, most of the time the additional parameters are independent of the level of damage or

\footnotetext{
* Corresponding author

Email address: cecile.oliver-leblond@dalembert.upmc.fr (C. Oliver-Leblond)
} 
stress - a proposal can be found for the non-local integral regularisation method where the internal length depends on the stress level [11] - even though the non-local interactions are expected to change according to the state of the medium.

A key question concerning non-local damage models of quasi-brittle materials is that of the physical origin of the non-locality and its proper incorporation in the continuum damage mechanics framework. This question has been earlier pointed out in several papers among which [12] for the non-local integral regularisation, [13] for the regularisation with a damage gradient and [14] for the regularisation with a strain gradient in $2 \mathrm{D}$.

An appropriate way to establish physical-based regularised damage models - and then to circumvent the aforementioned problems of the non-local material parameters - is to derive the constitutive law by means of homogenisation techniques. Therefore, the main objective of the present paper is to formulate and study a micro-mechanics based non-local damage model with strain gradient from the non-local estimates of Drugan and Willis [1]. The relevance of such a model, to which numerous works refer in the litterature, and its ability to capture the size effects due to nonlocality is investigated in this work.

The paper is organised as follows. First, from the non-local micro-mechanical analysis of Drugan and Willis, we derive the basic elements of the proposed macroscopic non-local damage model. The thermodynamic potential and the state laws are presented, as well as the damage criterion and the evolution laws. A careful use of the model is done in order to guarantee the positiveness of the thermodynamic potential and of the intrinsic dissipation. Then, we present the equilibrium equation and the boundary conditions which are classically deduced from the principle of virtual work. Finally, we establish the exact solution for the torsion of a cylindrical bar which obeys the non-local law. Size effects due to the non-local behaviour are illustrated. The impact of the non-local term on the damage evolution and the non-linear behaviour of the bar is also evaluated.

\section{Formulation of a micro-mechanics based strain gradient damage model}

\subsection{Drugan and Willis non-local elasticity model (1996)}

The present study is based on the micro-mechanical non-local constitutive equations established by Drugan and Willis [1]. Considering a class of two-phase composites with an isotropic and statistically uniform distribution of phases, these authors showed that the macroscopic strain energy depends on both the macroscopic strain state $E$ and the macroscopic strain gradient state $\nabla E$ :

$$
w(E, \nabla E)=\frac{1}{2} E \odot_{2}{ }^{(4)} \mathbb{C}^{h o m} \odot_{2} E+\frac{1}{2} \nabla E \odot_{3}{ }^{(6)} \mathbb{B}^{h o m} \odot_{3} \nabla E
$$

where $\odot_{n}$ is the $n^{\text {th }}$ tensor contraction between a tensor $\mathbb{A}$ of order greater than $n$ and a tensor $a$ of order $n$ such that $\left[\mathbb{A} \odot_{n} a\right]_{i, \ldots, j}=\mathbb{A}_{i, \ldots, j k, \ldots, l} a_{k, \ldots, l}$. For clarification, the energy potential given in equation $(1)$ can be rewritten in indicial notation : $w\left(E_{i j}, E_{i j, k}\right)=\frac{1}{2} E_{i j}{ }^{(4)} \mathbb{C}_{i j p q}^{h o m} E_{p q}+\frac{1}{2} E_{i j, k}{ }^{(6)} \mathbb{B}_{i j k p q r}^{h o m} E_{p q, r}$.

The energy potential (1) is continuously differentiable with respect to the macroscopic strain tensor $E$ and the macroscopic strain gradient tensor $\nabla E$. State laws can therefore be derived, thus providing the macroscopic stress tensor $\Sigma$ and the macroscopic double stress tensor $T$ :

$$
\Sigma=\frac{\partial w}{\partial E}={ }^{(4)} \mathbb{C}^{h o m} \odot_{2} E ; \quad T=\frac{\partial w}{\partial \nabla E}={ }^{(6)} \mathbb{B}^{h o m} \odot_{3} \nabla E
$$

If the structure is made of an isotropic matrix reinforced or weakened by a uniform dispersion of nonoverlapping identical isotropic spheres, both the $4^{\text {th }}$ order tensor ${ }^{(4)} \mathbb{C}^{\text {hom }}$ and the $6^{\text {th }}$ order tensor ${ }^{(6)} \mathbb{B}^{\text {hom }}$ can be explicitly constructed.

Let us consider a linear elastic matrix whose properties are the shear modulus $\mu_{0}$ and the bulk modulus $\kappa_{0}$. This matrix contains an uniform dispersion of non-overlapping identical spherical voids of volume concentration $c$ defined by: 


$$
c=\frac{4}{3} N \pi a^{3}
$$

where $a$ and $N$ respectively stand for the voids radius and the voids density i.e. the number of voids per unit of volume.

The classical Hashin-Shtrikhman upper bound provides the macroscopic stiffness tensor of the porous material:

$$
{ }^{(4)} \mathbb{C}^{\text {hom }}=3 \kappa^{\text {hom }(4)} \mathbb{J}+2 \mu^{\text {hom }}{ }^{(4)} \mathbb{K}
$$

where ${ }^{(4)} \mathbb{J}=I \otimes I / 3$ and ${ }^{(4)} \mathbb{K}={ }^{(4)} \mathbb{I}-{ }^{(4)} \mathbb{J}$ are the two isotropic $4^{\text {th }}$ order projectors, ${ }^{(4)} \mathbb{I}=I \otimes_{s} I$ is the symmetric $4^{\text {th }}$ order unit tensor, $I$ is the second order unit tensor, $\otimes$ is the tensor product and $\otimes_{s}$ the symmetric tensor product. The homogenised shear modulus and bulk modulus are respectively given by:

$$
\begin{gathered}
\mu^{\text {hom }}=\mu_{0} \frac{(1-c)\left(9 \kappa_{0}+8 \mu_{0}\right)}{9 \kappa_{0}+8 \mu_{0}+6 c\left(\kappa_{0}+2 \mu_{0}\right)} \\
\kappa^{\text {hom }}=\kappa_{0} \frac{4(1-c) \mu_{0}}{3 c \kappa_{0}+4 \mu_{0}}
\end{gathered}
$$

The isotropic $6^{\text {th }}$ order tensor obtained by Drugan and Willis reads:

$$
\begin{aligned}
{ }^{(6)} \mathbb{B}^{h o m} & =-\beta^{h o m}\left({ }^{(6)} \mathbb{K}_{2}+\frac{7}{4}{ }^{(6)} \mathbb{K}_{4}-\frac{7}{2}{ }^{(6)} \mathbb{K}_{6}\right)-\left(\frac{3}{4} \gamma^{h o m}+\frac{11}{4} \beta^{h o m}\right)\left({ }^{(6)} \mathbb{J}_{1}+{ }^{(6)} \mathbb{J}_{2}+{ }^{(6)} \mathbb{J}_{4}+{ }^{(6)} \mathbb{J}_{5}\right) \\
& \left.-\left(\frac{9}{4} \gamma^{h o m}+3 \beta^{h o m}\right)\left({ }^{(6)} \mathbb{J}_{3}+{ }^{(6)} \mathbb{J}_{6}\right)-\left(2 \gamma^{h o m}+\frac{3}{2} \beta^{h o m}\right)\left({ }^{(6)} \mathbb{J}_{7}+{ }^{(6)} \mathbb{J}_{8}\right)-\left(\frac{3}{2} \gamma^{h o m}+2 \beta^{h o m}\right)\right)^{(6)} \mathbb{J}_{9}
\end{aligned}
$$

where the $6^{\text {th }}$ order tensors ${ }^{(6)} \mathbb{K}_{n}$ for $n=1, \ldots, 6$ and ${ }^{(6)} \mathbb{J}_{m}$ for $m=1, \ldots, 9$ constitute an irreducible basis for the $6^{\text {th }}$ isotropic order tensors as proposed by [15]. Their expressions are given in Appendix A.

The non-local parameters $\beta^{\text {hom }}$ and $\gamma^{\text {hom }}$ depend on the porosity $c$ and the voids radius $a$ and are given by:

$$
\begin{aligned}
& \gamma^{\text {hom }}=4 c a^{2} \frac{(2-c)(1-c)^{2}}{5(1+2 c)} \mu_{0}^{2}\left(3 \kappa_{0}+4 \mu_{0}\right) \\
& \times \frac{5\left(3 \kappa_{0}+4 \mu_{0}\right)\left[21 \kappa_{0} \mu_{0}-2 \mu_{0}\left(3 \kappa_{0}+8 \mu_{0}\right)\right]-12(1-c) \kappa_{0} \mu_{0}\left(3 \kappa_{0}+\mu_{0}\right)}{21\left(3 c \kappa_{0}+4 \mu_{0}\right)\left[5 \mu_{0}\left(3 \kappa_{0}+4 \mu_{0}\right)-6(1-c) \mu_{0}\left(\kappa_{0}+2 \mu_{0}\right)\right]^{2}} \\
& \beta^{\text {hom }}=10 c a^{2} \frac{(2-c)(1-c)^{2}}{5(1+2 c)} \mu_{0} \frac{\left(3 \kappa_{0}+4 \mu_{0}\right)\left(3 \kappa_{0}+8 \mu_{0}\right)}{7\left[5\left(3 \kappa_{0}+4 \mu_{0}\right)-6(1-c)\left(\kappa_{0}+2 \mu_{0}\right)\right]^{2}}
\end{aligned}
$$

\subsection{Coupling with isotropic damage}

The formulation of a macroscopic strain gradient damage model requires the definition of the damage variable $d$. As Drugan and Willis's model is based on a micro-mechanical approach, with a micro-structure made of an isotropic matrix weakened by a random dispersion of non-overlapping spherical voids, the porosity $c$ appears as a natural candidate. To take into account $c_{0}$, the initial porosity of the material, it appears necessary to define the damage variable as:

$$
d=\frac{c-c_{0}}{1-c_{0}}
$$

The expressions of ${ }^{(4)} \mathbb{C}^{\text {hom }}(d)$ and ${ }^{(6)} \mathbb{B}^{\text {hom }}(d)$ are deduced from equations (4) and (7) by replacing the porosity $c$ by its expression as a function of $d$ : 


$$
c(d)=d\left(1-c_{0}\right)+c_{0}
$$

The porosity $c$ ranges from $c_{0}$ (virgin porous material) to 1 (ruined material) and is strictly increasing. Therefore, the damage variable ranges from 0 to 1 and is also strictly increasing.

It can be noted that a more common definition of the damage variable is obtained if the initial porosity $c_{0}$ tends towards 0 . In this case, the damage variable is equal to the porosity $c$ (see for example [16]).

The following rewriting of the free energy function (1) indicates its dependency on the internal variable $d:$

$$
w(E, \nabla E, d)=\frac{1}{2} E \odot_{2}{ }^{(4)} \mathbb{C}^{h o m}(d) \odot_{2} E+\frac{1}{2} \nabla E \odot_{3}{ }^{(6)} \mathbb{B}^{h o m}(d) \odot_{3} \nabla E
$$

where the tensors ${ }^{(4)} \mathbb{C}^{\text {hom }}$ and ${ }^{(6)} \mathbb{B}^{\text {hom }}$ are dependant on the damage variable $d=c$ according to equations (4), (5), (6), (7), (8) and (9).

State laws can therefore be rewritten to recall the macroscopic stress tensor $\Sigma$ and the macroscopic double stress tensor $T$ and to define the damage energy release rate $F_{d}$ :

$$
\begin{gathered}
\Sigma=\frac{\partial w}{\partial E}={ }^{(4)} \mathbb{C}^{h o m}(d) \odot_{2} E \\
T=\frac{\partial w}{\partial \nabla E}={ }^{(6)} \mathbb{B}^{h o m}(d) \odot_{3} \nabla E \\
F_{d}=-\frac{\partial w}{\partial d}=-\frac{1}{2} E \odot_{2}{ }^{(4)} \mathbb{C}_{, d}^{h o m}(d) \odot_{2} E-\frac{1}{2} \nabla E \odot_{3}{ }^{(6)} \mathbb{B}_{, d}^{h o m}(d) \odot_{3} \nabla E
\end{gathered}
$$

It can observed that the damage energy release rate consists in the sum of the classical local term and a non-local term due to the dependency of $\mathbb{B}^{\text {hom }}$ on $d$.

\subsection{Positiveness of the thermodynamic potential}

The free energy $w(E, \nabla E, d)$ must be positive for any arbitrary displacement field and damage level. The macroscopic strain tensor $E$ and the macroscopic strain gradient tensor $\nabla E$ being independent internal variables, both the local part of the free energy $w_{l}(E, d)=E \odot_{2}{ }^{(4)} \mathbb{C}^{h o m}(d) \odot_{2} E / 2$ and its non-local part $w_{n l}(\nabla E, d)=\nabla E \odot_{3}{ }^{(6)} \mathbb{B}^{h o m}(d) \odot_{3} \nabla E / 2$ must remain positive for any damage level. Therefore, the positiveness of the free energy implies that both the $4^{\text {th }}$ order tensor ${ }^{(4)} \mathbb{C}^{\text {hom }}(d)$ and the $6^{\text {th }}$ order tensor ${ }^{(6)} \mathbb{B}^{\text {hom }}(d)$ are positive definite for $d \in[0 ; 1]$.

The positiveness of the $4^{\text {th }}$ order tensor ${ }^{(4)} \mathbb{C}^{\text {hom }}(d)$ relies on those of $\kappa^{\text {hom }}(d)$ and $\mu^{\text {hom }}(d)$ which are ensured as long as $d \in[0 ; 1]$ (see equations (4), (6) and (5)).

The study of the positiveness of the $6^{\text {th }}$ order tensor ${ }^{(6)} \mathbb{B}^{h o m}(d)$ can be performed by taking advantage of the work of [15]. These authors showed that the previously introduced irreducible basis for isotropic $6^{\text {th }}$ order tensors allows the definition of the spherical part and the deviatoric part of a $3^{\text {rd }}$ order tensor such as $\nabla E$. It follows that the sign of the quadratic form associated with the $6^{\text {th }}$ order tensor can be easily analysed by noting that any isotropic $6^{\text {th }}$ order tensor ${ }^{(6)} \mathbb{A}$ can be decomposed as:

$$
{ }^{(6)} \mathbb{A}=\sum_{i=1}^{6} \alpha_{i}{ }^{(6)} \mathbb{K}_{i}+\sum_{i=1}^{9} \alpha_{i+6}{ }^{(6)} \mathbb{J}_{i}
$$

where $\alpha_{1}, \ldots, \alpha_{15}$ are the components of ${ }^{(6)} \mathbb{A}$ in the $\left(\mathbb{K}_{1}, \ldots, \mathbb{K}_{6}, \mathbb{J}_{1}, \ldots, \mathbb{J}_{9}\right)$ basis.

The positiveness of the $6^{\text {th }}$ order tensor ${ }^{(6)} \mathbb{A}$ is verified if and only if the following conditions are fulfilled:

$$
\begin{gathered}
0 \leq \alpha_{1} \\
0 \leq \alpha_{2} \\
0 \leq 2 \alpha_{3}+\alpha_{5} \\
0 \leq \alpha_{4}+2 \alpha_{6} \\
0 \leq \Psi_{1} ; \quad 0 \leq \Psi_{2} ; \quad 0 \leq \Psi_{3}
\end{gathered}
$$


where $\Psi_{1}, \Psi_{2}$ and $\Psi_{3}$ are the eigenvalues of matrix $\boldsymbol{\Psi}$ defined by:

$$
\boldsymbol{\Psi}=\left(\begin{array}{ccc}
3 \alpha_{7}+\alpha_{10}+\alpha_{13} & \alpha_{7}+3 \alpha_{10}+\alpha_{8} & \alpha_{7}+\alpha_{10}+3 \alpha_{13} \\
\alpha_{7}+3 \alpha_{10}+\alpha_{8} & \alpha_{8}+3 \alpha_{11}+\alpha_{14} & \alpha_{8}+\alpha_{11}+3 \alpha_{14} \\
\alpha_{7}+\alpha_{10}+3 \alpha_{13} & \alpha_{8}+\alpha_{11}+3 \alpha_{14} & \alpha_{9}+\alpha_{12}+3 \alpha_{15}
\end{array}\right)
$$

The coefficients $\alpha_{1}, \ldots, \alpha_{15}$ of the tensor ${ }^{(6)} \mathbb{B}^{\text {hom }}(d)$ being given in equation (7), the conditions for this tensor are reduced to:

$$
\begin{gathered}
0 \leq-\beta_{\text {hom }}(d) \\
0 \leq \frac{21}{4} \beta^{\text {hom }}(d) \\
0 \leq \beta^{\text {hom }}(d)-\gamma^{\text {hom }}(d) \\
0 \leq-7 \gamma_{\text {hom }}(d)-21 \beta^{\text {hom }}(d)+\sqrt{337 \gamma^{\text {hom }}(d)^{2}+1062 \gamma^{\text {hom }}(d) \beta^{\text {hom }}(d)+953 \beta^{\text {hom }}(d)^{2}} \\
0 \leq-7 \gamma^{\text {hom }}(d)-21 \beta^{\text {hom }}(d)-\sqrt{337 \gamma^{\text {hom }}(d)^{2}+1062 \gamma^{\text {hom }}(d) \beta^{\text {hom }}(d)+953 \beta^{\text {hom }}(d)^{2}}
\end{gathered}
$$

for $d \in[0 ; 1[$.
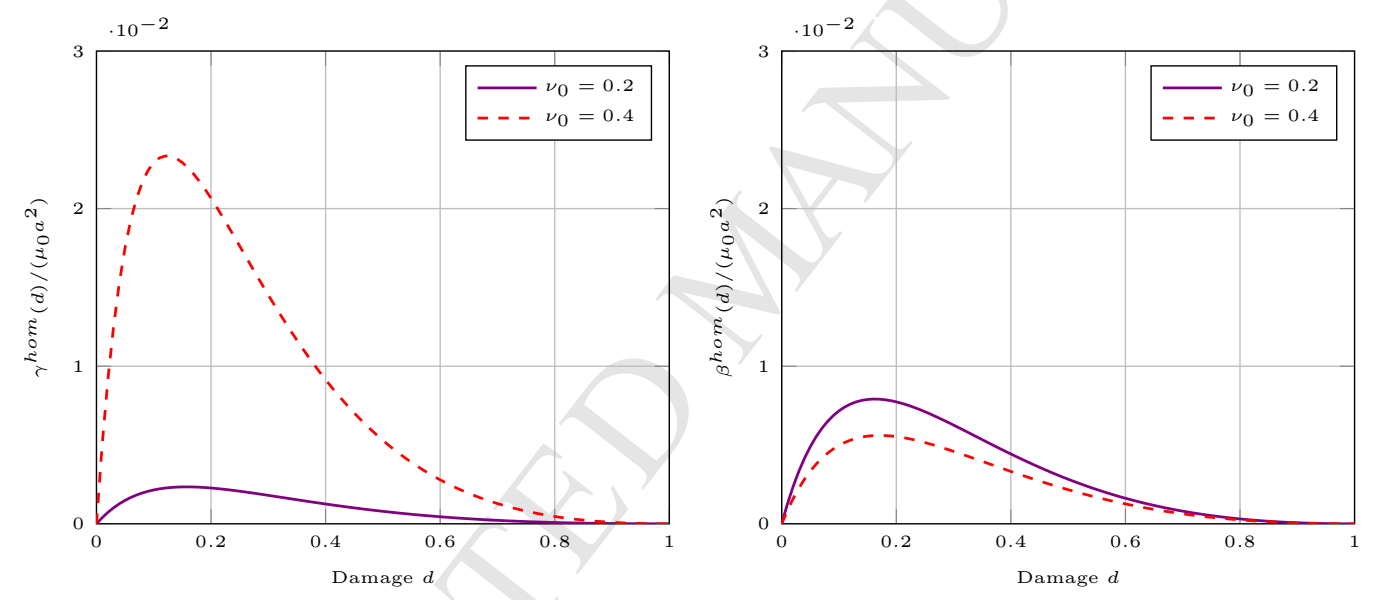

Figure 1: Variation of the non-local properties with damage $\left(E_{0}=30 \mathrm{GPa}, c_{0}=0\right)$

According to the variation of $\gamma^{\text {hom }}(d)$ and $\beta^{\text {hom }}(d)$ with $d$ (see figure 1), the first condition of equation (17) is violated. Moreover, the last three conditions of equation (17) cannot be simultaneously satisfied. Therefore, the positiveness of the $6^{\text {th }}$ order tensor ${ }^{(6)} \mathbb{B}^{h o m}(d)$ - and thus of the thermodynamical potential - cannot be achieved for an arbitrary loading. This lack of positiveness constitutes a strong limitation of the basic strain gradient elastic model proposed by [1] and therefore of the derived strain gradient damage model proposed in this paper. Indeed, certain loadings lead to a negative value of the thermodynamic potential. This is the case of the uni-dimensional traction of a bar for example.

In this paper, the torsion of a cylindrical bar will be later studied. In this case, only the second condition of equation (17) needs to be verified. We will show that the second deviatoric sub-part of the strain gradient tensor ${ }^{(6)} \mathbb{K}_{2} \odot_{3} \nabla E$ is equal to zero - then the first condition of (17) no longer raises any problem - and that the spherical sub-parts of the strain gradient tensor ${ }^{(6)} \mathbb{J}_{i} \odot_{3} \nabla E$ are all equal to zero for $i=1, \ldots, 9-$ then the last three conditions of (17) also no longer raise any problem.

\subsection{Damage criterion and evolution law}

The Clausius-Duhem inequality for an isothermal process involves a local term depending on the strain tensor rate $\dot{E}$ and the stress tensor $\Sigma$ and a non-local term depending on the strain gradient tensor rate $\nabla \dot{E}$ and the double stress tensor $T$ : 


$$
\mathcal{D}=\Sigma \odot_{2} \dot{E}+T \odot_{3} \nabla \dot{E}-\dot{w} \geq 0
$$

where $\mathcal{D}$ is the intrinsic dissipation.

The potential being continuously differentiable with respect to the macroscopic strain tensor $E$, the macroscopic strain gradient tensor $\nabla E$ and the damage variable $d,(18)$ reads:

$$
\mathcal{D}=\left(\Sigma-\frac{\partial w}{\partial E}\right) \odot_{2} \dot{E}+\left(T-\frac{\partial w}{\partial \nabla E}\right) \odot_{3} \nabla \dot{E}-\frac{\partial w}{\partial d} \dot{d} \geq 0
$$

According to the state laws (13), the intrinsic dissipation (19) can be rewritten in order to show its dependency on the damage mechanism:

$$
\mathcal{D}=F_{d} \dot{d}
$$

In order to ensure the positiveness of the intrinsic dissipation, the damage yield function must be convex with respect to $F_{d}$ and the corresponding damage domain must contain zero. Following [17] in the context of local damage models, the simplest form of the damage yield function is adopted:

$$
f\left(F_{d}, d\right)=F_{d}-G_{c}
$$

where $G_{c}$ is the critical damage energy.

The irreversible evolution of the damage variable is obtained by adopting the normality rule:

$$
\dot{d}=\dot{\lambda} \frac{\partial f}{\partial F_{d}}=\dot{\lambda} \quad \text { with } \quad \begin{cases}\dot{\lambda}=0 & \text { if } f<0 \text { or } \dot{f}<0 \\ \dot{\lambda}>0 & \text { if } f=0 \text { and } \dot{f}=0\end{cases}
$$

where $\dot{\lambda}$ is the damage multiplier.

Classically, the damage rate is deduced from the consistency condition, $\dot{f}=0$, which allows the determination of the damage multiplier. This leads to:

$$
\dot{d}=-\frac{\frac{\partial F_{d}}{\partial E} \odot_{2} \dot{E}+\frac{\partial F_{d}}{\partial \nabla E} \odot_{3} \nabla \dot{E}}{\frac{\partial F_{d}}{\partial d}}
$$

Given the expression (13) of the damage energy release rate $F_{d}$, we note the dependency of the damage rate on the first and second derivatives of tensors ${ }^{(4)} \mathbb{C}^{\text {hom }}(d)$ and ${ }^{(6)} \mathbb{B}^{\text {hom }}(d)$ with respect to $d$ :

$$
\dot{d}=-\frac{{ }^{(4)} \mathbb{C}_{, d}^{\text {hom }}(d) \odot_{2} E \odot_{2} \dot{E}+{ }^{(6)} \mathbb{B}_{, d}^{\text {hom }}(d) \odot_{3} \nabla E \odot_{3} \nabla \dot{E}}{\frac{1}{2} E \odot_{2}{ }^{(4)} \mathbb{C}_{, d d}^{\text {hom }}(d) \odot_{2} E+\frac{1}{2} \nabla E \odot_{3}{ }^{(6)} \mathbb{B}_{, d d}^{h o m}(d) \odot_{3} \nabla E}
$$

\subsection{Irreversibility of damage and positiveness of dissipation}

The stability of the damage evolution must be ensured. We suppose that the condition ensuring that the loading is causing damage, $f=0$ and $\dot{f}=0$, is fulfilled. It follows that:

$$
\frac{\partial F_{d}}{\partial E} \odot_{2} \dot{E}+\frac{\partial F_{d}}{\partial \nabla E} \odot_{3} \nabla \dot{E}>0
$$

According to the damage rate formulae during loading (see equations (23) and (24)), the irreversibility of damage $\dot{d}>0$ is then ensured if:

$$
\frac{1}{2} E \odot_{2}{ }^{(4)} \mathbb{C}_{, d d}^{h o m}(d) \odot_{2} E+\frac{1}{2} \nabla E \odot_{3}{ }^{(6)} \mathbb{B}_{, d d}^{h o m}(d) \odot_{3} \nabla E>0
$$

Consequently, both ${ }^{(4)} \mathbb{C}_{, d d}^{\text {hom }}(d)$ and ${ }^{(6)} \mathbb{B}_{, d d}^{\text {hom }}(d)$ must be positive definite, $E$ and $\nabla E$ being independent internal variables. 
Owing to the convexity of $\kappa^{h o m}(d)$ and $\mu^{\text {hom }}(d)$ with $d$ for $d \in[0 ; 1]$ (see equations (6) and (5)), it is readily proved that the positive definiteness of ${ }^{(4)} \mathbb{C}_{, d d}^{\text {hom }}(d)$ is ensured.

Concerning ${ }^{(6)} \mathbb{B}_{, d d}^{\text {hom }}(d)$ and in agreement with the conditions obtained in subsection 2.3 , the second derivative of the non-local $6^{\text {th }}$ order tensor ${ }^{(6)} \mathbb{B}^{\text {hom }}(d)$ is positive definite if and only if:

$$
\begin{gathered}
0<-\beta_{, d d}^{\text {hom }}(d) \\
0<\frac{21}{4} \beta_{, d d}^{\text {hom }}(d) \\
0<\beta_{, d d}^{\text {hom }}(d)-\gamma_{, d d}^{\text {hom }}(d) \\
0<-7 \gamma_{, d d}^{\text {hom }}(d)-21 \beta_{, d d}^{\text {hom }}(d) \pm \sqrt{337 \gamma_{, d d}^{\text {hom }}(d)^{2}+1062 \gamma_{, d d}^{\text {hom }}(d) \beta_{, d d}^{\text {hom }}(d)+953 \beta_{, d d}^{\text {hom }}(d)^{2}}
\end{gathered}
$$

Obviously, the first and the second conditions cannot both be verified and thus the irreversibility of damage is not ensured for any arbitrary loading. Furthermore, $\gamma^{\text {hom }}(d)$ and $\beta^{\text {hom }}(d)$ are not convex with respect to $d$ for $d \in[0 ; 1]$ (see figure 1) and thus it is impossible to find a loading which ensures (26).

To verify (26) for at least certain loadings such as the torsion test case, the studied material must have an initial porosity $c_{0}$ strictly greater than the inflection point $c_{\text {conv }}$ given by $\beta_{, d d}^{\text {hom }}\left(c_{\text {conv }}\right)=0$. Therefore, the use of this micromechanical non-local damage model is limited not only to a certain range of loadings but also to a certain class of materials.

Finally, according to equation (20), the positiveness of the intrinsic dissipation $\mathcal{D}$ is ensured if the damage rate $\dot{d}$ and the damage energy release rate $F_{d}$ are positive. The first condition $\dot{d} \geq 0$ requires that the second derivatives of tensors ${ }^{(4)} \mathbb{C}^{\text {hom }}(d)$ and ${ }^{(6)} \mathbb{B}^{\text {hom }}(d)$ with respect to $d$ are positive. The second condition $F_{d} \geq 0$ requires that the first derivatives of those tensors with respect to $d$ are negative. Given the expression of $\kappa^{\text {hom }}(d), \mu^{\text {hom }}(d), \gamma^{\text {hom }}(d)$ and $\beta^{\text {hom }}(d)$, the positiveness of the first derivatives of tensors ${ }^{(4)} \mathbb{C}^{\text {hom }}(d)$ and ${ }^{(6)} \mathbb{B}^{\text {hom }}(d)$ with respect to $d$ is satisfied when their second derivatives are positive. Therefore, the solution presented above to ensure the irreversibility of damage is necessary and sufficient to ensure the positiveness of the intrinsic dissipation.

Remark: as demonstrated before, the positiveness of the intrinsic dissipation depends on the convexity of the non-local functions $\gamma^{\text {hom }}(d)$ and $\beta^{\text {hom }}(d)$. According to [1], the non-local parameters $\gamma^{\text {hom }}(d)$ and $\beta^{\text {hom }}(d)$ depend on a radial integral of the two-point correlation function $h(r)$. Willis [18] showed that this two-point correlation function must satisfy the relation:

$$
h(r)=\frac{P(r)-d^{2}}{d(1-d)}
$$

where $d$ is the damage variable and $P(r)$ is the probability that two points separated by the radial coordinate $r$ fall within the same phase (inclusion or matrix). An analytical approximation of this two-point correlation function is proposed in [19]. This approximation is then used in [1] to analytically compute the non-local parameters $\gamma^{\text {hom }}(d)$ and $\beta^{\text {hom }}(d)$ used in this study. However, other choices can be made on the approximation of the two-point correlation function $h(r)$ (see for instance the work of [20]) and thus it may be worth considering further works in order to formulate an approximation of the two-point correlation function which guarantees the positiveness of the intrinsic dissipation.

\subsection{Link between the damage variable and the voids' radius}

According to equations (5), (6), (8), (9) and (11), the material parameters of the non-local damage model are the elastic properties of matrices $\mu_{0}$ and $\kappa_{0}$, the initial porosity $c_{0}$ and the radius of the voids $a$. However, depending on the type of damage mechanism that takes place at the micro-level, the radius of the voids can be a function $a(d)$ of the damage variable.

Therefore, the non-local tensor ${ }^{(6)} \mathbb{B}^{\text {hom }}(d)$ will be chosen accordingly to the type of degradation that occurs at the micro-level: 
- Nucleation of the micro-voids: The radius of the voids is a fixed material parameter $a(d)=a$ and an evolution of the damage variable is understood as an increase of the void density $N$. The $6^{\text {th }}$ order homogenised tensor reads:

$$
{ }^{(6)} \mathbb{B}_{*}^{\text {hom }}(d)={ }^{(6)} \mathbb{B}_{\text {nuc }}^{\text {hom }}(d)=a^{2(6)} \overline{\mathbb{B}}^{\text {hom }}(d)
$$

where ${ }^{(6)} \overline{\mathbb{B}}^{\text {hom }}(d)={ }^{(6)} \mathbb{B}^{\text {hom }}(d) / a(d)^{2}$.

- Growth of the micro-voids: The void density $N$ is a fixed material parameter and an evolution of the damage variable is understood as an uniform growth of the micro-voids. Therefore, the radius of the voids is given by $a(d)=(3 c(d) / 4 N \pi)^{1 / 3}$. The $6^{\text {th }}$ order homogenised tensor then reads:

$$
{ }^{(6)} \mathbb{B}_{*}^{h o m}(d)={ }^{(6)} \mathbb{B}_{g r}^{h o m}(d)=\left(\frac{3 c(d)}{4 N \pi}\right)^{2 / 3}{ }^{(6)} \overline{\mathbb{B}}^{h o m}(d)
$$

Remark: in the case of the growth of the voids, the average distance between the nearest voids appears in the definition of the non-local $6^{\text {th }}$ order homogenised tensor. Indeed, this mean distance is equal to $\left(\frac{3}{4 N \pi}\right)^{1 / 3} \Gamma(4 / 3)$ as demonstrated in [21] and reminded in Appendix B.
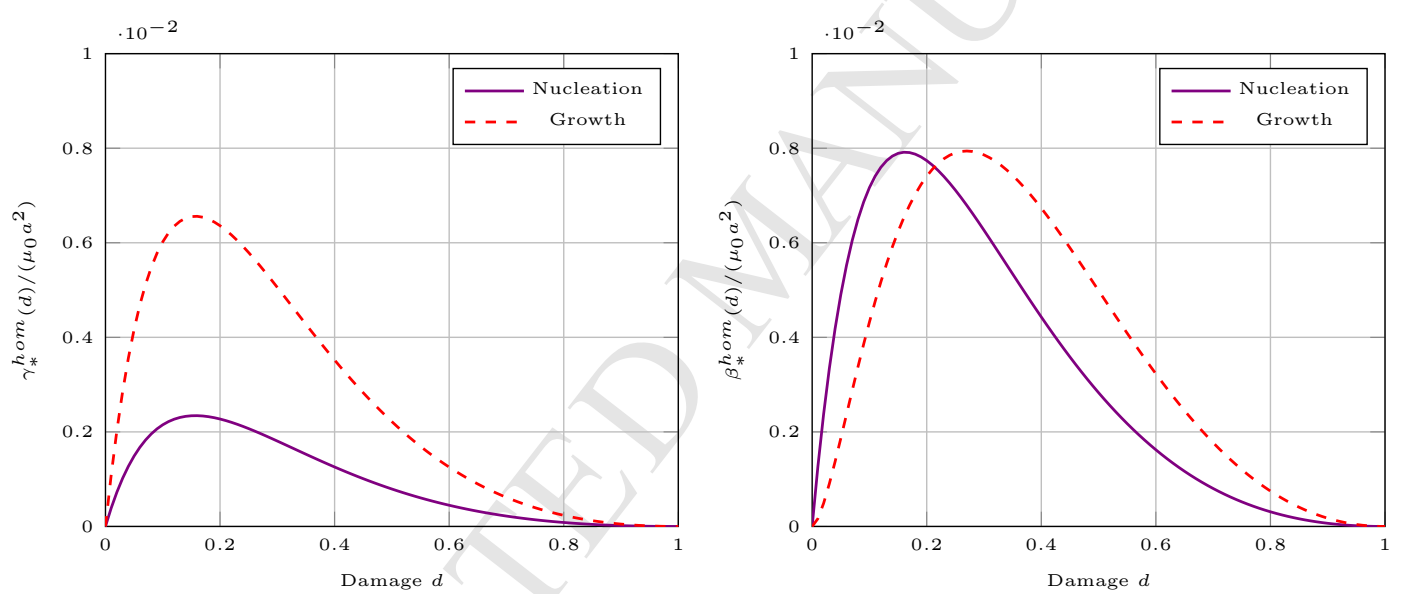

Figure 2: Variation of the non-local properties with the damage for each type of damage mechanism $\left(E_{0}=30 \mathrm{GPa}, \nu_{0}=0.21\right.$, $a=1 \mu \mathrm{m}$ and $N=5.110^{16}$ )

The type of damage mechanisms only influences the evolution of the non-local damage parameters with $d$. Indeed, the voids' radius does not appear in the definition of the local part of the model. As seen on figure 2 , the evolution of the non-local parameters with damage in the voids growth case and in the nucleation case are quite similar and thus the same conclusions can be made in terms of positive definiteness, damage irreversibility and thermodynamic admissibility. Since the maximum of the function is reached at different damage levels according to the damage mechanisms, the definition of the initial porosity $c_{0}$ is dependent on the damage mechanisms.

\subsection{Equilibrium and boundary conditions}

The introduction of higher order gradients of the displacement field $U$ in the formulation of a damage model leads to non-classical equilibrium equation and boundary conditions. In [22, 23], those equations are obtained for elasticity with a second displacement gradient.

In order to derive the equilibrium equation and the boundary conditions associated with the strain gradient elasticity, the principle of virtual work is written to show its dependency on the symmetric strain tensor $E=(\nabla U+U \nabla) / 2$ and the strain gradient $\nabla E$ : 


$$
\int_{V}\left(\Sigma \odot_{2} \delta E+T \odot_{3} \delta \nabla E\right) d V=\int_{V} f \cdot \delta U d V+\int_{S}(t \cdot \delta U+r \cdot(D \delta U)) d S
$$

where the stress tensor $\Sigma$ and the double stress tensor $T$ balance the body force $f$, the surface traction $t$ and the surface double force traction $r$.

Following [22], the gradient of the displacement variation on the boundary surface which is decomposed into a surface gradient and a normal gradient is introduced:

$$
\nabla \delta U=\stackrel{s}{\nabla} \delta U+n D \delta U ; \quad \stackrel{s}{\nabla}=\left({ }^{(2)} \mathbb{I}-n \otimes n\right) \cdot \nabla ; \quad D=n \cdot \nabla
$$

where $n$ is a unit vector orthogonal to surface $S$

Applying (31) Gauss's divergence theorem on the volume integrals and Stokes's surface divergence theorem on the closed smooth surface integral (see definition in [24]), one gets the equilibrium equation:

$$
\nabla \nabla T-\nabla \Sigma=f \quad \text { in } V
$$

and the boundary conditions:

$$
\begin{gathered}
(\Sigma-\nabla T-\stackrel{s}{\nabla}(T \cdot n)) \cdot n+\stackrel{s}{\nabla} n T \cdot n \cdot n=t \quad \text { or } \quad U=\bar{U} \quad \text { on } S \\
T \cdot n \cdot n=r \quad \text { or } \quad D U=\overline{D U} \quad \text { on } S
\end{gathered}
$$

where $\bar{U}$ is the applied displacement and $\overline{D U}$ is the applied normal gradient of the displacement.

The distinction between a natural condition and an essential condition is generalised as proposed by [25]. Four kinds of boundary conditions may be applied on each surface combining $t$-natural or $U$-essential conditions and $r$-natural or $D U$-essential conditions.

\subsection{Local integration of the strain gradient damage model}

In order to capture the evolution of the state variables, let us introduce an iterative scheme which aims at determining the mechanical state $\left\{E_{n+1}, \nabla E_{n+1}, d_{n+1}, \Sigma_{n+1}, T_{n+1}\right\}$ at time $t_{n+1}$ from the mechanical state $\left\{E_{n}, \nabla E_{n}, d_{n}, \Sigma_{n}, T_{n}\right\}$ at time $t_{n}$ and the loading increments $\Delta E$ and $\Delta \nabla E$.

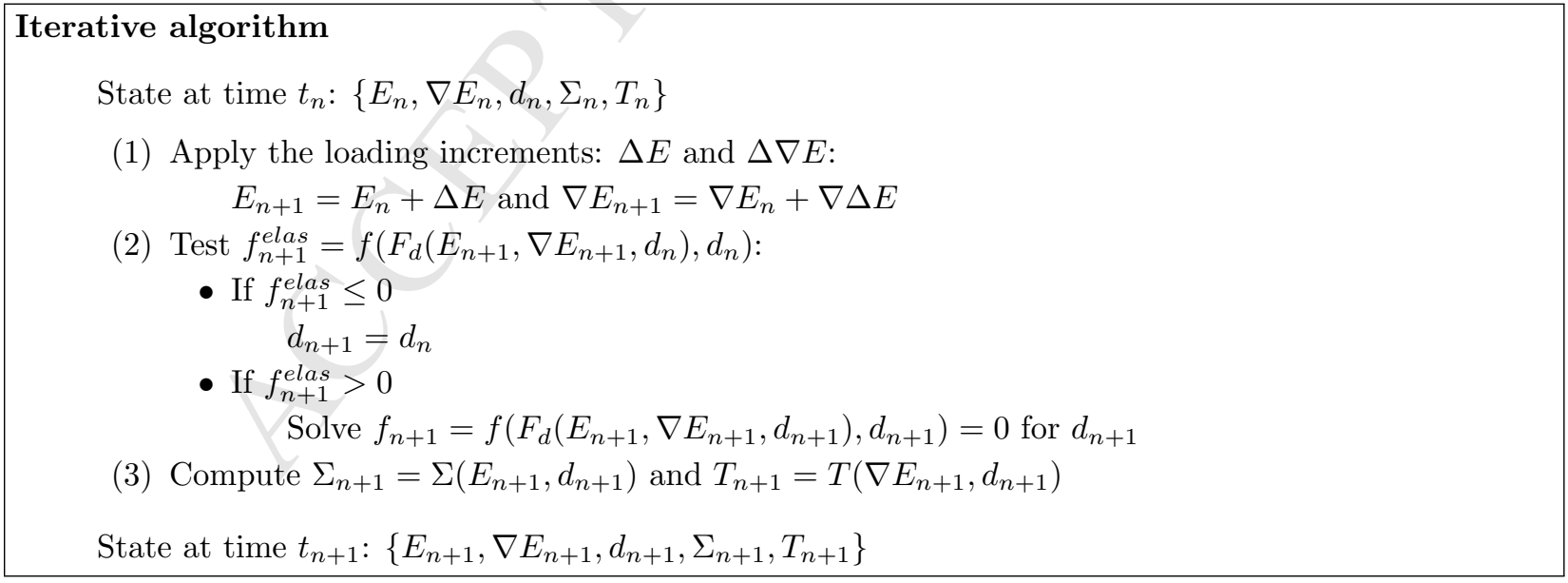




\section{Exact elastic solution for the torsion of a cylindrical bar at fixed damage level}

The constitutive equations with strain gradient presented in the previous section are now applied to the study of a cylindrical bar of radius $R$ and height $h$ under torsion (see figure 3). Following [26], the boundary conditions read:

a. Fixed displacements on base $S_{0}$;

b. Applied twist angle $\alpha$ on base $S_{h}$;

c. No surface double force traction on $S_{0}$ and $S_{h}$;

d. No surface force traction and double surface force traction on lateral surface $S_{l}$;

e. No body forces.

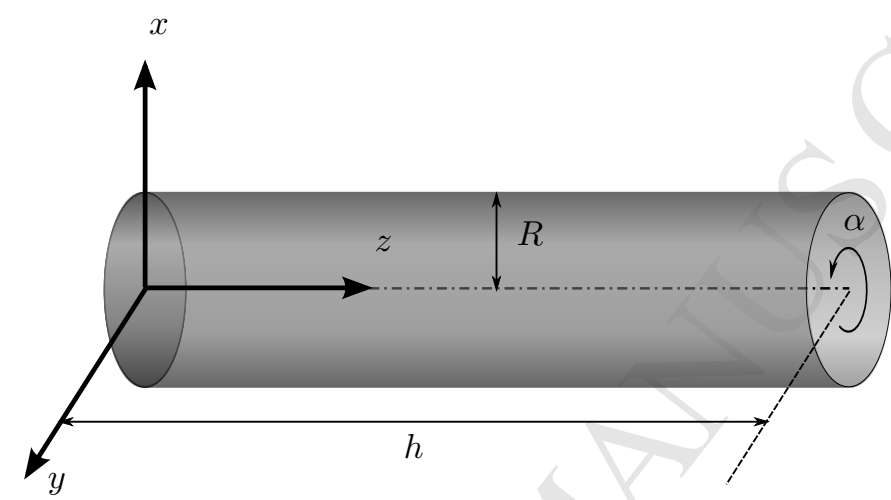

Figure 3: Cylindrical bar under torsion: geometry, loading and coordinate system

Torsion experiments on various materials - such as metals [27], polymeric foams [28] or bones [29] -have been used to highlight size effects and confirm the need to introduce non-local effects through a characteristic length linked to the micro-structure.

\subsection{Analytical solution for the elastic problem at fixed damage level}

In order to construct the elastic non-local solution to the torsion problem, the damage is first fixed to a value $d_{0}$ strictly greater than zero.

The displacement field is assumed to be axisymmetric:

$$
U_{x}=-y \theta(z) ; \quad U_{y}=x \theta(z) ; \quad U_{z}=0
$$

where $\theta$ is the rotation angle of the bar around the $z$-axis.

The non-vanishing components of the strain tensor and the strain gradient tensor are then:

$$
\begin{gathered}
E_{x z}=E_{z x}=-\frac{y}{2} \frac{d \theta}{d z} ; \quad E_{y z}=E_{z y}=\frac{x}{2} \frac{d \theta}{d z} \\
E_{x z, y}=E_{z x, y}=-\frac{1}{2} \frac{d \theta}{d z} ; \quad E_{y z, x}=E_{z y, x}=\frac{1}{2} \frac{d \theta}{d z} \\
E_{x z, z}=E_{z x, z}=-\frac{y}{2} \frac{d^{2} \theta}{d z^{2}} ; \quad E_{y z, z}=E_{z y, z}=\frac{x}{2} \frac{d^{2} \theta}{d z^{2}}
\end{gathered}
$$

In the case of torsion, $\operatorname{Tr}(E)=0$ and thus the strain energy density can be rewritten as:

$$
w(E, \nabla E)=\mu^{h o m}\left(d_{0}\right) E \odot_{2} E+\frac{7}{4} \beta^{h o m}\left(d_{0}\right) \nabla E \odot_{3} \nabla E
$$


It can be readily verified that the positive definitiveness is ensured for $d_{0} \in[0 ; 1]$. Therefore, the model can be applied to the study of torsion at fixed damage level without any restriction.

We can observe that (38) has a form similar to that of many energy potentials proposed in the literature for elasticity models with strain gradient $[30,31]$ :

$$
w(E, \nabla E)=\mu^{h o m}\left(d_{0}\right) E \odot_{2} E+l\left(d_{0}\right)^{2} \mu^{h o m}\left(d_{0}\right) \nabla E \odot_{3} \nabla E
$$

where $l$ is usually a phenomenological internal length. Here, the internal length is deduced from a micromechanical analysis for the torsion problem by noticing that the dimension of $\beta^{\text {hom }}\left(d_{0}\right)$ is a pressure times length squared:

$$
l\left(d_{0}\right)=\sqrt{\frac{7 \beta^{\text {hom }}\left(d_{0}\right)}{4 \mu^{\text {hom }}\left(d_{0}\right)}}
$$

This internal length is proportional to the radius of the voids $a$, and depends on the Poisson's ratio of the matrix $\nu_{0}$, the initial porosity $c_{0}$ and on the damage level $d_{0}$.

According to (2), the non-vanishing components of the stress tensor and the double stress tensor are:

$$
\Sigma_{x z}=\Sigma_{z x}=-\mu^{h o m}\left(d_{0}\right) y \frac{d \theta}{d z} ; \quad \Sigma_{y z}=\Sigma_{z y}=\mu^{h o m}\left(d_{0}\right) x \frac{d \theta}{d z}
$$

and:

$$
\begin{aligned}
& T_{x x x}=T_{z z x}=\frac{3 y}{4}\left(\gamma^{\text {hom }}\left(d_{0}\right)+2 \beta^{\text {hom }}\left(d_{0}\right)\right) \frac{d^{2} \theta}{d z^{2}} ; \quad T_{y y y}=T_{z z y}=-\frac{3 x}{4}\left(\gamma^{\text {hom }}\left(d_{0}\right)+2 \beta^{\text {hom }}\left(d_{0}\right)\right) \frac{d^{2} \theta}{d z^{2}} \\
& T_{x x y}=-\frac{3 x}{4} \gamma^{h o m}\left(d_{0}\right) \frac{d^{2} \theta}{d z^{2}} ; \quad T_{y y x}=\frac{3 y}{4} \gamma^{h o m}\left(d_{0}\right) \frac{d^{2} \theta}{d z^{2}} \\
& T_{x y x}=T_{y x x}=-\frac{3 x}{4} \beta^{h o m}\left(d_{0}\right) \frac{d^{2} \theta}{d z^{2}} ; \quad T_{x y y}=T_{y x y}=\frac{3 y}{4} \beta^{h o m}\left(d_{0}\right) \frac{d^{2} \theta}{d z^{2}} \\
& T_{x z y}=T_{z x y}=-\frac{7}{4} \beta^{\text {hom }}\left(d_{0}\right) \frac{d \theta}{d z} ; \quad T_{y z x}=T_{z y x}=\frac{7}{4} \beta^{\text {hom }}\left(d_{0}\right) \frac{d \theta}{d z} \\
& T_{x z z}=T_{z x z}=\frac{y}{2} \beta^{h o m}\left(d_{0}\right) \frac{d^{2} \theta}{d z^{2}} ; \quad T_{y z z}=T_{z y z}=-\frac{x}{2} \beta^{h o m}\left(d_{0}\right) \frac{d^{2} \theta}{d z^{2}}
\end{aligned}
$$

The equilibrium equation (33) reduces to:

$$
\mu^{h o m}\left(d_{0}\right) \frac{d^{2} \theta}{d z^{2}}+\beta^{h o m}\left(d_{0}\right) \frac{d^{4} \theta}{d z^{4}}=0
$$

The form of the solution to (43) is:

$$
\theta(z)=c_{1} \cos \omega z+c_{2} \sin \omega z+c_{3} z+c_{4}
$$

where $\omega=\sqrt{\frac{2 \mu^{h o m}\left(d_{0}\right)}{\beta^{h o m}\left(d_{0}\right)}}$ and $c_{1}, \ldots, c_{4}$ are constants which will be obtained from the boundary conditions.

Applying boundary conditions (c), (a) and (b), we obtain:

$$
\frac{d^{2} \theta}{d z^{2}}(0)=0 ; \quad \frac{d^{2} \theta}{d z^{2}}(h)=0 ; \quad \theta(0)=0 ; \quad \theta(h)=\alpha
$$

The solution for the twist angle, which also satisfies boundary conditions (d) on lateral surface $S_{l}$, is thus the same as for the local problem, namely:

$$
\theta(z)=\frac{\alpha z}{h}
$$


The resultant torque in the bar, noted $M$, is obtained by applying the virtual work principle on cross section $S(z)$ :

$$
M(z) \frac{d \theta(z)}{d z}=\frac{1}{2} \int_{S(z)}\left(\Sigma \odot_{2} E+T \odot_{3} \nabla E\right) d S
$$

It follows that:

$$
M(\alpha)=M_{0}\left(1+4 \frac{l^{2}}{R^{2}}\right)
$$

where $M_{0}$ is the resultant torque for the local problem:

$$
M_{0}(\alpha)=\mu^{h o m} \frac{\pi R^{4}}{2} \frac{\alpha}{h}
$$

\subsection{Illustration of the results at fixed damage level}

We here study the evolution of the normalised torque $M / M_{0}$ with the normalised radius $R / a$ for a matrix of Young modulus $E_{0}=50 \mathrm{GPa}$ and Poisson ratio $\nu_{0}=0.2$ weakened by voids. The initial porosity of the material is $c_{0}$ and the damage level is $d_{0}=0$. Therefore, following equation (40), the normalised internal length is $l\left(d_{0}\right) / a=0.14$.

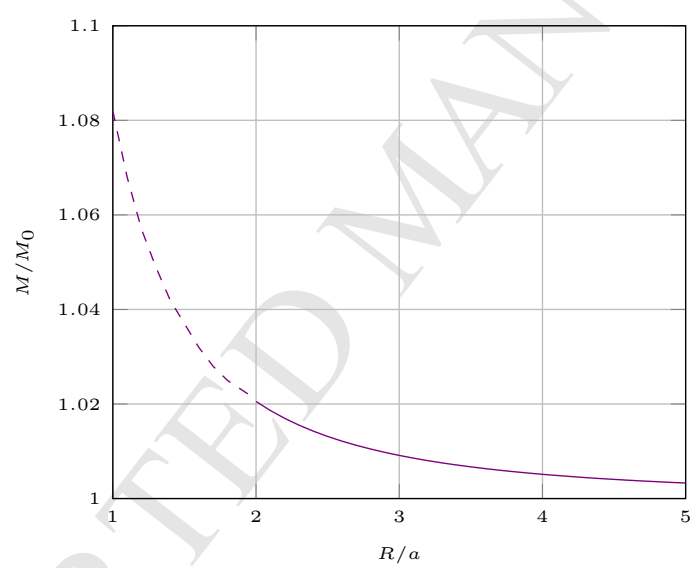

Figure 4: Effect of the normalised radius on the torsional rigidity of the cylindrical bar at fixed damage level

We can observe on figure 4 that the influence of the non-local term is negligible - less than $1 \%$ - if the radius of the cylinder $R$ is more than three times the radius $a$ of the voids. Those results are in agreement with the work of Drugan and Willis [1] who showed that the representative volume element size - defined as the minimum ensemble for which the non-local term produces a negligible correction to the local term is approximately four radius for any type of inclusions and for all volume fractions.

On figure 4, the non-local effect increases when the radius of the cylinder $R$ decreases towards zero. However, those results are clearly non-physical if $R$ is not superior to at least two times the radius of the inclusion $a$.

\section{Axisymmetric solution for the torsion of a damageable cylindrical bar}

\subsection{Analytical solution for the damage problem}

In this part, the torsion problem is studied in the context of an evolving damage. To this end, let us assume that the displacement field is the same as the one obtained for an elastic case at fixed damage level: 


$$
U_{x}=-y \frac{\alpha z}{h} ; \quad U_{y}=x \frac{\alpha z}{h} ; \quad U_{z}=0
$$

The non-vanishing components of the strain tensor and the gradient of strain tensor are:

$$
\begin{gathered}
E_{x z}=E_{z x}=-\frac{y}{2} \frac{\alpha}{h} ; \quad E_{y z}=E_{z y}=\frac{x}{2} \frac{\alpha}{h} \\
E_{x z, y}=E_{z x, y}=-\frac{\alpha}{2 h} ; \quad E_{y z, x}=E_{z y, x}=\frac{\alpha}{2 h}
\end{gathered}
$$

Similarly to the form (38) for the fixed damage case, the free energy function for the torsional problem can be rewritten in the case of an evolving damage as:

$$
w(E, \nabla E, d)=\mu^{h o m}(d) E \odot_{2} E+\frac{7}{4} \beta^{h o m}(d) \nabla E \odot_{3} \nabla E
$$

The positiveness of this reduced potential is unconditionally ensured for $d \in[0 ; 1]$.

The internal length introduced in equation (40) is now a function of the damage:

$$
l(d)=\sqrt{\frac{7 \beta^{h o m}(d)}{4 \mu^{h o m}(d)}}
$$

According to (13), the non-vanishing components of the stress tensor and the double stress tensor are:

$$
\begin{gathered}
\Sigma_{x z}=\Sigma_{z x}=-\mu^{h o m}(d) y \frac{\alpha}{h} ; \quad \Sigma_{y z}=\Sigma_{z y}=\mu^{h o m}(d) x \frac{\alpha}{h} \\
T_{x z y}=T_{z x y}=-\frac{7}{4} \beta^{h o m}(d) \frac{\alpha}{h} ; \quad T_{y z x}=T_{z y x}=\frac{7}{4} \beta^{h o m}(d) \frac{\alpha}{h}
\end{gathered}
$$

The shear stress $\Sigma^{s h}$ and the shear hyper-stress $T^{s h}$ are obtained with:

$$
\Sigma^{s h}=\mu^{h o m}(d) r \frac{\alpha}{h} ; \quad T^{s h}=\frac{7}{4} \beta^{h o m}(d) \frac{\alpha}{h}
$$

where $r=\sqrt{x^{2}+y^{2}}$ is the radial distance to the z-axis.

The damage energy release rate $F_{d}$ takes the form:

$$
F_{d}=-\mu_{, d}^{h o m}(d) E \odot_{2} E-\frac{7}{4} \beta_{, d}^{h o m}(d) \nabla E \odot_{3} \nabla E
$$

As seen before in subsection 2.5, the damage irreversibility and the positiveness of the intrinsic dissipation are ensured if $F_{d}$ is positive and if (26) is verified. Given the evolution of the local parameter with the damage, the conditions on the local part of $F_{d}$ are verified. However, an initial porosity $c_{0}$ has to be defined in order the verify the conditions on the non-local part of $F_{d}$.

The damage criterion is:

$$
-\frac{1}{2}\left(\mu_{, d}^{h o m}(d) r^{2}+\frac{7}{2} \beta_{, d}^{h o m}(d)\right)\left(\frac{\alpha}{h}\right)^{2}-G_{c} \leq 0
$$

From this damage criterion, it is readily seen that $d$ is function of the imposed twist angle $\alpha$ and of the radial position $r$.

Remark: in order to find a solution to the non-local damage problem, the displacement field has been assumed to be in the form of the non-local elastic one obtained in subsection 3.1. The damage field obtained with (59) ensures the validation of this hypothesis. Indeed, the equilibrium and the boundary conditions are satisfied because $d$ is independent of the angular position and the z-position. 
Finally, the evolution of the torque with the twist angle $M(\alpha)$ is obtained by computing the following integral:

$$
M(\alpha)=2 \pi \frac{\alpha}{h} \int_{0}^{R} \mu^{h o m}(d(r, \alpha))\left(1+2 \frac{l(d(r, \alpha))^{2}}{r^{4}}\right) r^{3} d r
$$

The computation of the torque $M_{0}(\alpha)$ predicted by the local model is also recalled:

$$
M_{0}(\alpha)=2 \pi \frac{\alpha}{h} \int_{0}^{R} \mu^{h o m}(d(r, \alpha)) r^{3} d r
$$

In the case of the local model, it is possible to analytically find the relation between the twist angle and the resulting torque [32]. However, given the expression of $\beta_{, d}^{\text {hom }}(d)$, the integral (60) does not admit an analytical expression and thus the evolution of the non-local torque with the twist angle will be obtained numerically.

\subsection{Illustration of the results: mechanical field and damage distribution}

The following results have been obtained for a typical material whose characteristics are presented in table 1. As seen on figure 2, the choice of damage mechanism only has a small influence on the evolution of $\beta^{\text {hom }}(d)$ and thus we restrict our analysis to the case of nucleation. Therefore, the radius of the voids is a fixed material parameter.

Taking into account the results obtained at fixed damage level (see figure 4), the dimensions of the cylinder are set to $R=2 \mu \mathrm{m}$ and $h=10 R$ in order to observe the influence of non-local term on the torsional behaviour.

\begin{tabular}{|c|c|c|c|}
\hline Symbol & Physical meaning & Value & Unit \\
\hline \hline$E_{0}$ & Matrix Young Modulus & 50 & $\mathrm{GPa}$ \\
\hline$\nu_{0}$ & Matrix Poisson ratio & 0.2 & - \\
\hline \hline$a$ & Radius of the voids & 1 & $\mu \mathrm{m}$ \\
\hline$c_{0}$ & Initial porosity & 0.35 & - \\
\hline \hline$G_{c}$ & Critical energy & 0.001 & $\mathrm{~J} . \mathrm{m}^{-2}$ \\
\hline
\end{tabular}

Table 1: Properties of the material

Remark: the typical material studied here is supposed to be initially porous. Therefore, the initial elastic properties of the homogenised material $\mu^{\text {hom }}(0)$ and $\kappa^{\text {hom }}(0)$ when the material is sound are not equal to the elastic properties of the matrix $\mu_{0}$ and $\kappa_{0}$. However, if $\mu^{\text {hom }}(0)$ and $\kappa^{\text {hom }}(0)$ have been experimentally obtained by studying the initial porous material, $\mu_{0}$ and $\kappa_{0}$ can be derived from eq (5) and (6) with $d=0$ or $c=c_{0}$.

The evolution of the internal length with the damage (see figure 5) is explained by its dependency on statistical factor $H=\int_{0}^{\infty} h(r) r d r$ (given in [1]) where $h(r)$ is the two-point correlation function which indicates the probability that two points separated by a distance $r$ are in the same phase. Obviously, when $d \rightarrow 1$ then $c(d) \rightarrow 1$ and thus the probability of finding two points in the matrix phase approaches 0 .

The evolution of the damage is obtained by implementing the iterative algorithm presented in subsection 2.8. When the damage reaches 1 on radius $R$, one can pursue the computation by removing the totally damaged zone and calculating the behaviour on the inner part of the cylinder which is not fully damaged as proposed by [32]. The associated critical twist angle is:

$$
\alpha_{c r}=\frac{h}{R} \sqrt{-\frac{2 G_{c}}{\mu_{, d}^{\text {hom }}(1)}}
$$




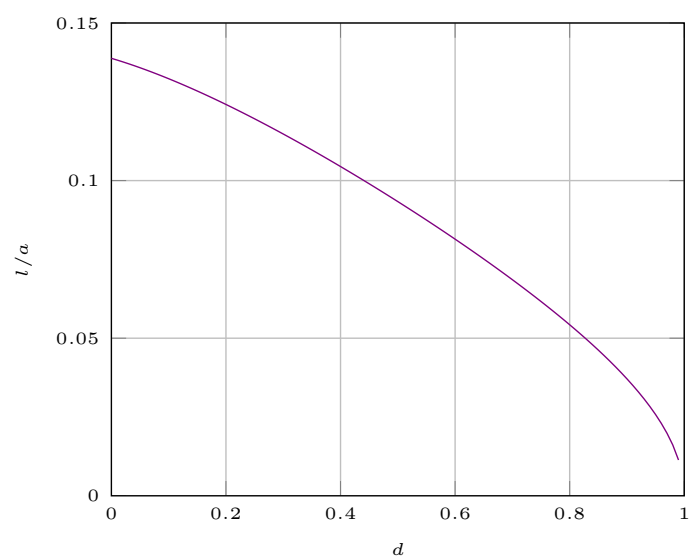

Figure 5: Evolution of the torsional internal length with the damage

For the set of parameters given in table 1, the critical twist angle is $4.8810^{-6} \mathrm{rad}$.

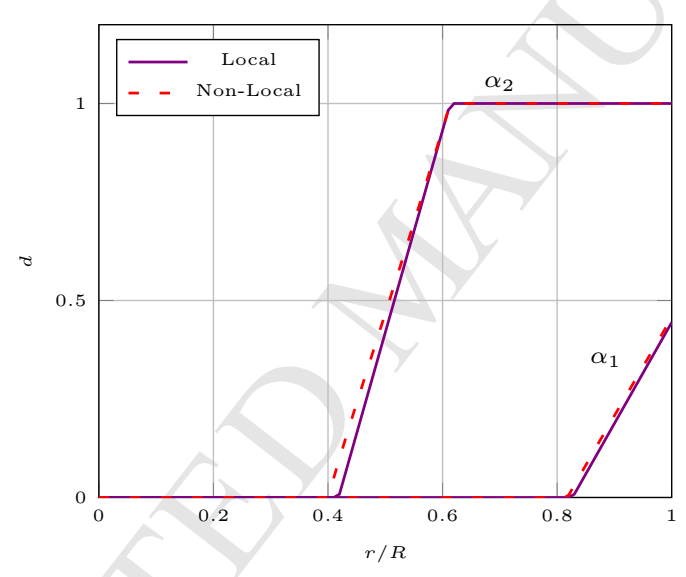

Figure 6: Distribution of the damage along the radius for $\alpha_{1}=410^{-6} \mathrm{rad}$ and $\alpha_{2}=810^{-6} \mathrm{rad}$

The distribution of damage along the radius for two level of loadings $\alpha_{1}=410^{-6} \mathrm{rad}$ and $\alpha_{2}=810^{-6} \mathrm{rad}$ is given on figure 6 . As expected, the damaged area is initially formed outside of the cylinder and moves inwards in the form of an annular ring. For $\alpha_{1}=810^{-6} \mathrm{rad}$, which is greater than the critical value, the cylinder is composed of a sound inner part, a ruined outer part and a damaged part in between. According to the damage criterion (see equation (59)), the evolution of the damage is more influenced by the non-local parameter for small radii.

The distribution of the shear stress $\Sigma^{s h}$ and the shear hyper-stress $T^{s h}$ along the radius for two level of loadings $\alpha_{1}=410^{-6} \mathrm{rad}$ and $\alpha_{2}=810^{-6} \mathrm{rad}$ is given on figure 7 . The shear stress is influenced by the non-local term only through the damage variable. Therefore, in the inner undamaged part of the cylinder, the non-local term has no impact on the shear stress distribution. After the damage initiation radius, the shear stress decreases with the radius and reaches zero if the twist angle is greater than the critical value. Because the addition of the non-local term leads to a slight advance in the initiation of the damage, the decrease of the shear stress with the radius appears sooner in the non-local case. The shear hyper-stress is not equal to zero in the inner undamaged part of the cylinder because we take into account an initial porosity. The shear hyper-stress is proportional to non-local parameter $\beta^{\text {hom }}$ and thus decreases with the evolution of damage.

The evolution of the torque with the twist angle is given on figure 8. According to the torque formula 

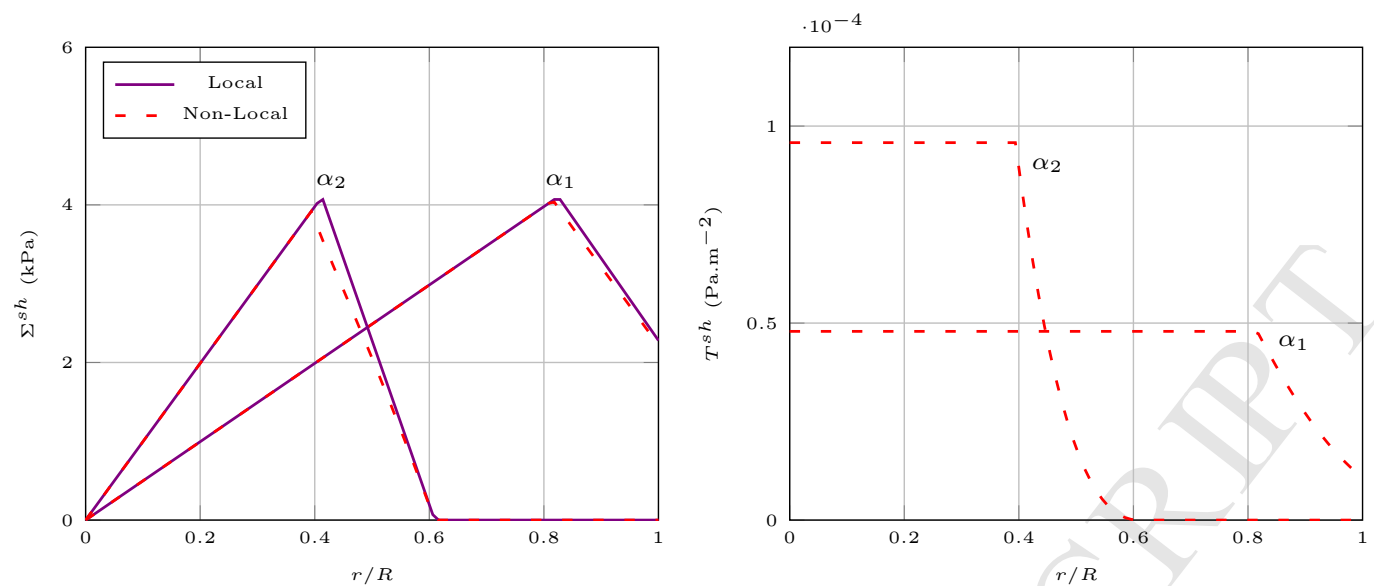

Figure 7: Distribution of the shear stress (left) and the shear hyper-stress (right) along the radius for $\alpha_{1}=410^{-6} \mathrm{rad}$ and $\alpha_{2}=810^{-6} \mathrm{rad}$
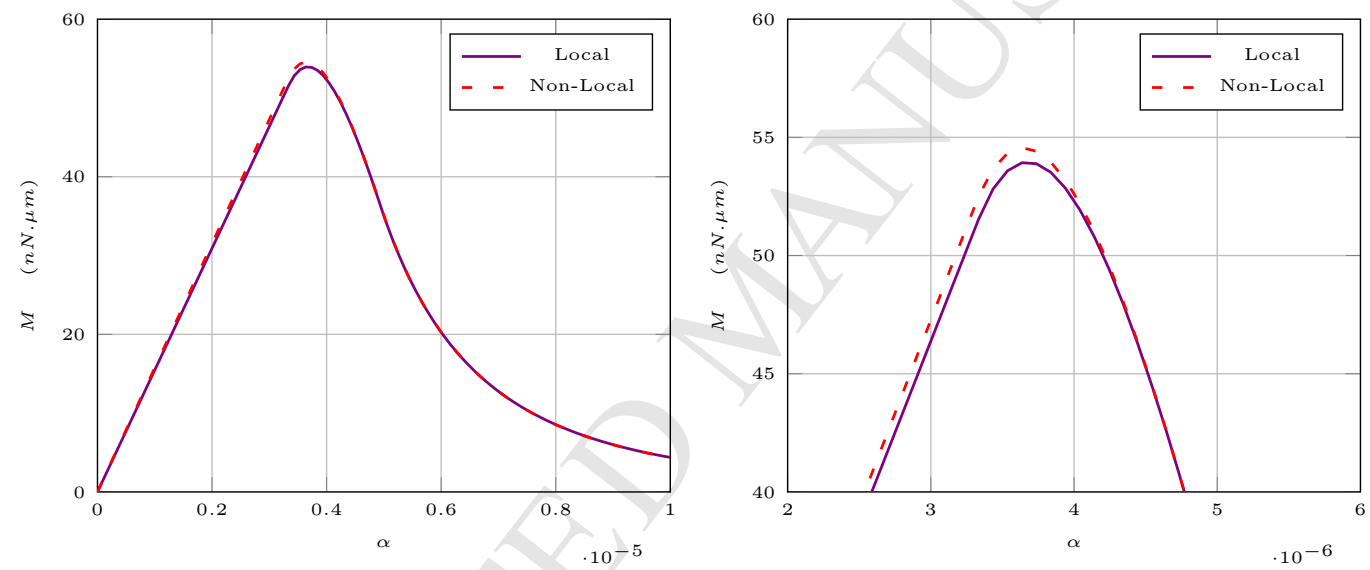

Figure 8: Variation of the torque with the twist angle (left: complete response, right: zoom on the peak)

(see equation (60)), the influence of the non-local modelling intervenes not only through the addition of a non-local term but also through the damage evolution. Although the non-local effect is hardly noticeable, the presence of the non-local term impacts the complete torque evolution, even in the elastic phase, because the initial porosity is not equal to zero. The non-local term also implies an advance in the initiation of the damage and therefore on the peak.

In order to study the size effect, the evolution of the maximal normalised torque $\max (M) / \max \left(M_{0}\right)$ with the normalised radius $R / a$ is plotted on figure 9 . As observed for the case at fixed damage level (on figure 4 ), the non-local effect disappears if $R / a$ increases.

\section{Conclusions}

In this paper, we present a non-local damage model whose formulation is based on the micro-mechanical results obtained by Drugan and Willis [1] for a matrix containing an uniform dispersion of non-overlapping identical isotropic spherical voids. The thermodynamic potential obtained is the sum of the classical local part and a non-local part depending on the strain gradient. This second part does not introduce a simple characteristic length but a $6^{\text {th }}$ order tensor depending on the properties of the elastic matrix and on the radius of the voids. 


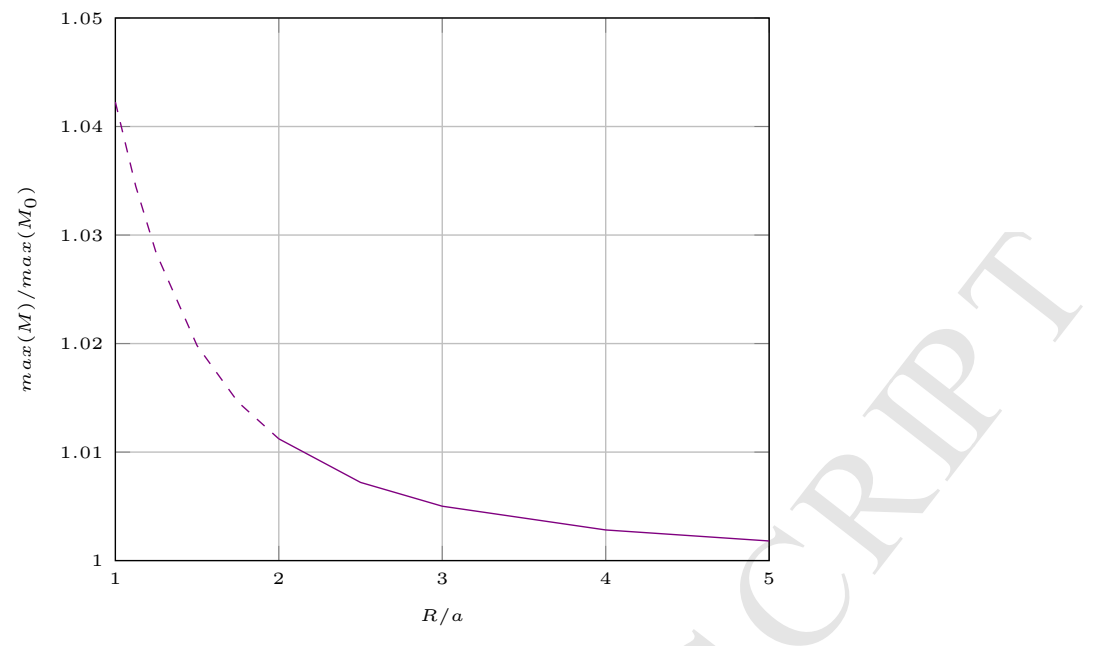

Figure 9: Effect of the normalised radius on the torsional behaviour of the cylindrical bar

A general study of the non-local model shows the lack of positiveness of the thermodynamic potential and of the intrinsic dissipation. The strain gradient model is thus applied to the study of the torsion of a cylindrical bar to ensure the positiveness of the thermodynamic potential. Moreover, the damage variable is defined as a function of the porosity and a non-zero initial porosity is needed to guarantee the positiveness of the intrinsic dissipation.

The study of a cylindrical bar under torsion is first performed at a fixed damage level to demonstrate that the non-local elastic displacement solution is the same as the local elastic displacement solution. The size effect, caused by the presence of the strain gradient term, is studied. The non-local damage model is then applied to the study of the torsion of a cylindrical bar. The impact of the non-local term on the damage evolution and the mechanical field is found to be quite small. Indeed, the equivalent torsional characteristic length obtained with this strain gradient damage model is of the order of the voids' radius. Thus, the higherorder effects due to the introduction of the strain gradient can be expected to come into play when the length scale of the strain field becomes comparable to the micro-structural length scale [33, 23]. To further explore the role of those higher-order effects, the authors believe that the comparison with experiments on microspecimen of porous material subjected to damage would be necessary. Those experiments could follow the ones proposed by Lakes [28] for microelasticity.

For further developments, it is necessary to work on the micro-mechanical analysis in order to define a thermodynamic potential which remains positive definite for any loading. For example, it would be interesting to formulate and study a regularised damage model derived from the non-local exact constitutive equations proposed by Drugan [34] - which are not gradient approximated as in the present paper. These equations may ensure the positiveness of the thermodynamic potential and the intrinsic dissipation.

Once those thermodynamical properties are ensured, an analysis of the bifurcation and stability of the non-local damage model may be performed following for instance the work of [35].

\section{Acknowledgements}

The authors would like to acknowledge Dr Kim Pham for fruitful and technical discussions at the beginning of the present study.

\section{References}

[1] W. Drugan, J. Willis, A micromechanics-based nonlocal constitutive equation and estimates of representative volume element size for elastic composites, J. Mech. Phys. Solids 44 (1996) 497-524. 
[2] G. Pijaudier-Cabot, Z. Bažant, Nonlocal damage theory, Journal of Engineering Mechanics 1113 (1987) $1512-1533$.

[3] R. Peerlings, R. de Borst, W. Brekelmans, I. de Vree, J.H.P. ans Spee, Some observations on localisation in non-local and gradient damage models, Eur. J. Mech. A/Solids 15 (6) (1996) 937-953.

[4] E. Kuhl, E. Ramm, Simulation of strain localization with gradient enhanced damage models, Computational Materials Sciences 16 (2000) 176-185.

[5] M. Frémond, B. Nedjar, Damage, gradient of damage and principle of virtual work, Int. J. Solids Struct. 33 (8) (1996) $1083-1103$.

[6] E. Lorentz, S. Andrieux, A variational formulation of nonlocal damage models, Int. J. Plas. 15 (1999) $119-138$.

[7] K. Pham, J.-J. Marigo, From the onset of damage to rupture: construction of responses with damage localization for a general class of gradient damage models, Continuum Mech. Thermodyn. 25 (2013) 147-171.

[8] W. Zhou, J. Zhao, Y. Liu, Q. Yang, Simulation of localization failure with strain-gradient-enhanced damage mechanics, Int. J. Numer. Anal. Meth. Geomech. 26 (2002) 793-813.

[9] P. Walsh, Fracture of plain concrete, Indian Concrete Journal 46 (11).

[10] Z. Bazant, M. Jirasek, Nonlocal integral formulations of plasticity and damage: survey of progress, Journal of Engineering Mechanics 128 (11) (2002) 1119-1149.

[11] C. Giry, F. Dufour, J. Mazars, Stress based nonlocal damage model, Int. J. Solids Struct. 48 (25-26) (2011) 3431-3443.

[12] Z. Bazant, Nonlocal damage theory based on micromechanics of crack interactions, Journal of Engineering Mechanics 120 (3) (1994) 593-617.

[13] S. Andrieux, M. Joussemet, E. Lorentz, A class of constitutive relations with internal variable derivatives: derivation from homogenization, C.R. Acad. Sci. 323 (1996) 629-636.

[14] J. Li, A micromechanics-based strain gradient damage model for fracture prediction of brittle materials - part i: Homogenization methodology and constitutive relations, Int. J. Solids Struct. 48 (2011) 3336-3345.

[15] V. Monchiet, G. Bonnet, On the inversion of non symmetric sixth-order isotropic tensors and conditions of positiveness of third-order tensor valued quadratic functions, Mechanics Research Communications 38 (2011) 326-329.

[16] V. Pensee, D. Kondo, L. Dormieux, Micromechanical analysis of anisotropic damage in brittle materials, Journal of Engineering Mechanics 128 (2002) 889-897.

[17] J. Marigo, Modeling of brittle and fatigue damage for elastic material by growth of micro- voids, Eng. Fract. Mech. 21 (4) (1985) 861-874.

[18] J. Willis, Elasticity theory of composites, Hopkins, H.G., Sewell, M.J. (Eds.), Mechanics of Solids: The R. Hill 60th Anniversary Volume Pergamon Press, Oxford (653-686).

[19] K. Markov, J. Willis, On the two-point correlation function for dispersions of nonoverlapping spheres, Mathematical Models and Methods in Applied Sciences 8 (2) (1998) 359-377.

[20] I. Monetto, W. Drugan, A micromechanics-based nonlocal constitutive equation for elastic composites containing randomly oriented spheroidal heterogeneities, Journal of the Mechanics and Physics of Solids 52 (2) (2004) 359-393.

[21] S. Chandrasekhar, Stochastic problems in physics and astronomy, Reviews of modern physics 15 (1) (1943) 1-89, p. 86.

[22] R. Mindlin, Second gradient of strain and surface tension in linear elasticity, Int. J. Solids Struct. 1 (1965) $417-438$.

[23] N. Fleck, J. Hutchinson, Strain gradient plasticity, Advances in Applied Mechanics 33 (1997) $295-361$.

[24] L. Brand, Vector and tensor analysis, John Wiley \& Sons (1947) p. 222.

[25] F. Dell'Isola, G. Sciarra, S. Vidoli, Generalized hooke's law for isotropic second gradient materials, Proc. R. Soc. A 465 (2107) (2009) 2177-2196.

[26] D. Lam, F. Yang, A. Chong, J. Wang, P. Tong, Experiments and theory in strain gradient elasticity, J. Mech. Phys. Solids 51 (2003) 1477-1508.

[27] N. Fleck, G. Muller, M. Ashby, J. Hutchinson, Strain gradient plasticity: theory and experiment, Acta Metallurgica et Materialia 42 (2) (1994) 475-487.

[28] R. Lakes, Size effects and micromechanics of a porous solid, Journal of Materials Science 18 (1983) 2572-2580.

[29] J. Yang, R. Lakes, Transient study of couple stress effects in compact bone: torsion, Journal of biomechanical engineering 103 (4) (1981) 275-279.

[30] B. Altan, E. Aifantis, On the structure of the mode iii crack tip in gradient elasticity, Scripta Metallurgica et Materialia 26 (2) (1992) 319-324.

[31] S. Akarapu, H. Zbib, Numerical analysis of plane cracks in strain-gradient elastic materials, International Journal of Fracture 141 (2006) 403-430.

[32] E. Lanoye, L. Dormieux, D. Kondo, A micromechanical-based damage analysis of a cylindrical bar under torsion: theoretical results and verification by finite elements computations, Theoretical and Applied Fracture Mechanics 74 (2014) $116-125$

[33] R. Mindlin, Micro-structure in linear elasticity, Arch. Ration. Mech. Anal. 16 (1964) 51-78.

[34] W. Drugan, Two exact micromechanics-based nonlocal constitutive equations for random linear elastic composite materials, J. Mech. Phys. Solids 51 (2003) 1745-1772.

[35] K. Pham, J.-J. Marigo, C. Maurini, The issues of the uniqueness and the stability of the homogeneous response in uniaxial tests with gradient damage models, Journal of the Mechanics and Physics of Solids 59 (2011) 1163-1190.

\section{Appendix A. Basis for isotropic sixth-order tensors}

The $6^{\text {th }}$ order tensors ${ }^{(6)} \mathbb{K}_{n}$ for $n=1, \ldots, 6$ and ${ }^{(6)} \mathbb{J}_{m}$ for $m=1, \ldots, 9$ proposed by [15] are given by: 


$$
\begin{aligned}
& \left.{ }^{\left({ }^{6}\right)} \mathbb{K}_{1}\right]_{i j k p q r}=\frac{1}{6}\left(\delta_{i p} \delta_{j q} \delta_{k r}+\delta_{i r} \delta_{j p} \delta_{k q}+\delta_{i q} \delta_{j r} \delta_{k p}-\delta_{i r} \delta_{j q} \delta_{k p}-\delta_{i p} \delta_{j r} \delta_{k q}-\delta_{i q} \delta_{j p} \delta_{k r}\right) \\
& \left.{ }^{\left({ }^{6}\right)} \mathbb{K}_{2}\right]_{i j k p q r}=\frac{1}{6}\left(\delta_{i p} \delta_{j q} \delta_{k r}+\delta_{i r} \delta_{j p} \delta_{k q}+\delta_{i q} \delta_{j r} \delta_{k p}+\delta_{i r} \delta_{j q} \delta_{k p}+\delta_{i p} \delta_{j r} \delta_{k q}+\delta_{i q} \delta_{j p} \delta_{k r}\right) \\
& -\frac{1}{15}\left(\delta_{i j} \delta_{k r} \delta_{p q}+\delta_{i j} \delta_{k p} \delta_{q r}+\delta_{i j} \delta_{k q} \delta_{p r}+\delta_{i j} \delta_{j r} \delta_{p q}\right. \\
& \left.+\delta_{i j} \delta_{j p} \delta_{q r}+\delta_{i k} \delta_{j q} \delta_{p r}+\delta_{j k} \delta_{i r} \delta_{p q}+\delta_{j k} \delta_{i p} \delta_{q r}+\delta_{j k} \delta_{i q} \delta_{p r}\right) \\
& {\left[{ }^{(6)} \mathbb{K}_{3}\right]_{i j k p q r}=\frac{1}{6}\left(\delta_{i j} \delta_{j r} \delta_{p q}+\delta_{i j} \delta_{j p} \delta_{q r}-\delta_{j k} \delta_{i r} \delta_{p q}-\delta_{j k} \delta_{i p} \delta_{q r}\right)} \\
& +\frac{1}{3}\left(\delta_{i p} \delta_{j q} \delta_{k r}+\delta_{i r} \delta_{j q} \delta_{k p}+\delta_{j k} \delta_{i q} \delta_{p r}-\delta_{i q} \delta_{j r} \delta_{k p}-\delta_{i q} \delta_{j p} \delta_{k r}-\delta_{i k} \delta_{j q} \delta_{p r}\right) \\
& {\left[{ }^{(6)} \mathbb{K}_{4}\right]_{i j k p q r}=\frac{1}{6}\left(\delta_{i j} \delta_{j p} \delta_{q r}+\delta_{i k} \delta_{j q} \delta_{p r}-\delta_{j k} \delta_{i p} \delta_{q r}-\delta_{j k} \delta_{i q} \delta_{p r}\right)} \\
& +\frac{1}{3}\left(\delta_{i q} \delta_{j r} \delta_{k p}+\delta_{i p} \delta_{j r} \delta_{k q}+\delta_{j k} \delta_{i r} \delta_{p q}-\delta_{i r} \delta_{j p} \delta_{k q}-\delta_{i r} \delta_{j q} \delta_{k p}-\delta_{i j} \delta_{j r} \delta_{p q}\right) \\
& {\left[{ }^{(6)} \mathbb{K}_{5}\right]_{i j k p q r}=\frac{1}{6}\left(\delta_{i j} \delta_{k r} \delta_{p q}+\delta_{i j} \delta_{k p} \delta_{q r}-\delta_{j k} \delta_{i r} \delta_{p q}-\delta_{j k} \delta_{i p} \delta_{q r}\right)} \\
& +\frac{1}{3}\left(\delta_{i r} \delta_{j p} \delta_{k q}+\delta_{i p} \delta_{j r} \delta_{k q}+\delta_{j k} \delta_{i q} \delta_{p r}-\delta_{i q} \delta_{j r} \delta_{k p}-\delta_{i q} \delta_{j p} \delta_{k r}-\delta_{i j} \delta_{k q} \delta_{p r}\right) \\
& {\left[{ }^{(6)} \mathbb{K}_{6}\right]_{i j k p q r}=\frac{1}{6}\left(\delta_{i j} \delta_{k p} \delta_{q r}+\delta_{i j} \delta_{k q} \delta_{p r}-\delta_{j k} \delta_{i p} \delta_{q r}-\delta_{j k} \delta_{i q} \delta_{p r}\right)} \\
& +\frac{1}{3}\left(\delta_{i p} \delta_{j q} \delta_{k r}+\delta_{i q} \delta_{j p} \delta_{k r}+\delta_{j k} \delta_{i r} \delta_{p q}-\delta_{i r} \delta_{j p} \delta_{k q}-\delta_{i r} \delta_{j q} \delta_{k p}-\delta_{i j} \delta_{k r} \delta_{p q}\right) \\
& \left.{ }^{(6)} \mathbb{J}_{1}\right]_{i j k p q r}=\frac{1}{10}\left(4 \delta_{j k} \delta_{i p} \delta_{q r}-\delta_{j k} \delta_{i r} \delta_{p q}-\delta_{j k} \delta_{i q} \delta_{p r}\right) \\
& {\left[{ }^{(6)} \mathbb{J}_{2}\right]_{i j k p q r}=\frac{1}{10}\left(4 \delta_{j k} \delta_{i q} \delta_{p r}-\delta_{j k} \delta_{i p} \delta_{q r}-\delta_{j k} \delta_{i r} \delta_{p q}\right)} \\
& {\left[{ }^{(6)} \mathbb{J}_{3}\right]_{i j k p q r}=\frac{1}{10}\left(4 \delta_{j k} \delta_{i r} \delta_{p q}-\delta_{j k} \delta_{i q} \delta_{p r}-\delta_{j k} \delta_{i p} \delta_{q r}\right)} \\
& \left.{ }^{\left({ }^{6)} \mathbb{J}_{4}\right.}\right]_{i j k p q r}=\frac{1}{10}\left(4 \delta_{i j} \delta_{j p} \delta_{q r}-\delta_{i j} \delta_{j r} \delta_{p q}-\delta_{i k} \delta_{j q} \delta_{p r}\right) \\
& \left.{ }^{\left({ }^{(6)} J_{5}\right.}\right]_{i j k p q r}=\frac{1}{10}\left(4 \delta_{i k} \delta_{j q} \delta_{p r}-\delta_{i j} \delta_{j r} \delta_{p q}-\delta_{i j} \delta_{j p} \delta_{q r}\right) \\
& \left.{ }^{\left({ }^{(6)}\right.} \pi_{6}\right]_{i j k p q r}=\frac{1}{10}\left(4 \delta_{i j} \delta_{j r} \delta_{p q}-\delta_{i j} \delta_{j p} \delta_{q r}-\delta_{i k} \delta_{j q} \delta_{p r}\right) \\
& {\left[{ }^{(6)} \mathbb{J}_{7}\right]_{i j k p q r}=\frac{1}{10}\left(4 \delta_{i j} \delta_{k p} \delta_{q r}-\delta_{i j} \delta_{k r} \delta_{p q}-\delta_{i j} \delta_{k q} \delta_{p r}\right)} \\
& {\left[{ }^{(6)} \mathbb{J}_{8}\right]_{i j k p q r}=\frac{1}{10}\left(4 \delta_{i j} \delta_{k q} \delta_{p r}-\delta_{i j} \delta_{k r} \delta_{p q}-\delta_{i j} \delta_{k p} \delta_{q r}\right)} \\
& {\left[{ }^{(6)} \mathbb{J}_{9}\right]_{i j k p q r}=\frac{1}{10}\left(4 \delta_{i j} \delta_{k r} \delta_{p q}-\delta_{i j} \delta_{k p} \delta_{q r}-\delta_{i j} \delta_{k q} \delta_{p r}\right)}
\end{aligned}
$$

\section{Appendix B. Mean distance between nearest neighbours}

We are looking for the mean distance between nearest neighbours of a a set of points distributed according to a Poisson distribution in $3 \mathrm{D}$ with a density $N$. Therefore, the number of points in a volume $V$ also follows a Poisson distribution of mean $N V$. The probability of finding $k$ points in the volume $V$ is:

$$
P(V, k)=\frac{(N V)^{k} e^{-N V}}{k !}
$$


The nearest neighbour of a random point is situated between $r$ and $r+d r$ if there is no point before $r$ and at least one between $r$ and $r+d r$. The probability is:

$$
\begin{aligned}
d P(r) & =P\left(4 N \pi r^{3} / 3,0\right)\left(1-P\left(4 N \pi r^{2} d r, 0\right)\right) \\
& =e^{-4 N \pi r^{3} / 3}\left(1-e^{-4 N \pi r^{2} d r}\right) \\
& \simeq 4 N \pi e^{-4 N \pi r^{3} / 3} r^{2} d r
\end{aligned}
$$

The mean distance between nearest neighbours is given by:

$$
l=\int_{0}^{\infty} r d P(r)=\int_{0}^{\infty} 4 N \pi e^{-4 N \pi r^{3} / 3} r^{2} d r
$$

Performing the change of variable $s=4 N \pi r^{3} / 3$ we obtain:

$$
l=\left(\frac{3}{4 N \pi}\right)^{1 / 3} \int_{0}^{\infty} s^{1 / 3} e^{-s} d s=\left(\frac{3}{4 N \pi}\right)^{1 / 3} \Gamma(4 / 3)
$$




\section{Highlights}

A micro-mechanics based strain gradient damage model: formulation and solution for the torsion of a cylindrical bar

- We propose a theoretical formulation for an isotropic damage model with strain gradient.

- We derive the basic elements of the proposed macroscopic non-local damage model from the non-local micro-mechanical analysis of Drugan and Willis (1996).

- We study the size effect, caused by the presence of the strain gradient term, on the torsion problem. 